\title{
1973 Membership Directory
}

\author{
(Corrected to February 1, 1973) \\ Permission for Commercial Use Must Be Obtained From \\ the NAALD Executive Secretary
}

The following list contains, in addition to NALLD members, the names of libraries that are subscribers. If you want to support NALLD, and your library is not listed, have them place an order.

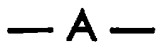

MS DOLORES ABALLI, St. Mary's College, Notre Dame, IN 46566

H. J. ABOYAN, Ridgefield School District, 610 Cedar Grove Road, Broomall, PA 19008

MS SUDIE M. ADAMS, Dept. of Spanish, Baylor U., Box 159, Waco, TX 76703 MR. CHARLES F. ADAMSON, 821 Osceola Ave., Saint Paul Academy, St. Paul, .MI 55105

MS NELLIE AGOSTINO, Chrm. of the Lang. Dept., East Hart HS, 777 Burnside Ave., East Hartford, CT 06117

MS HELEN C. AGUERA, Federal City College, 425 Second St., NW, Washington, DC 20001

JOHN A. AHERN, Westchester Community College, 75 Grasslands Road, Valhalla, NY 10595

H. F. AIKENS, Director of the Lang. Lab., Dalhousie University, Halifax, Nova Scotia, Canada

AIMAV, Secretariat: Av Georges Bergmann, 109-1050 Bruxelles, BELGIUM

LANG. LAB., U. of Alberta, Edmonton, Alberta, Canada, Att. Ms W. C. Deurloo

DIRECTOR OF THE LANG. LAB., Allegheny College, Meadville, PA 16335

- HOWARD B. ALTMAN, Dept. of Germanic Lang., U. of Washington, Seattle, WA 98105

LEE ALTMAN, Lang. Lab. Dir., San Francisco State Col., 1600 Holloway, San Francisco, CA 94132

EMMANUEL D'AMONVILLE, Chmn. of the For. Lang. Dept., Thayer Academy, Braintree, MA 02185

SISTER ROSE TERESA AMOR, Chmn. Span. Dept., Molloy Catholic College, Rockville Centre, NY 11570

THE DIRECTOR, An Teanglann, University College, Galway, Ireland CHM. OF MOD. LANG. DEPT., Andrews U., Berrien Springs, MI 49104

MS FRANCOISE APPEL, Chapman College, 333 North Glassell St., Orange, CA 92666

ARIZONA STATE U., Library - Periodicals, Tempe, AZ 85281

ETILVIA ARJONA-Profesora Laboratorio de Lenguas, Estafeta Universitaria, Panama, Rep. de Panama 
MS ELIZABETH ARMISTEAD, Lees-McRae College, Box 618, Banner Elk, NC 28604

JEAN GUY ASSELIN, Supervisor, Carleton University Lang. Lab., Colonel By Drive, Ottawa 1, Ontario, Canada

MRS. GEORGE M. ATKINSON, 1529 Denver Ave., Apt. 10, Kansas City, MO 64127

CECIL ATTIG, Lang. Lab. Supervisor, California State U., Fullerton, CA 92631

G. E. AUCOIN, Box 760, Sydney, Nova Scotia, Canada

MR. JEAN Y. AUDIGIER, 315 Horizon Way, Pacifica, CA 94044

PROF. GILLES E. AUGER, Nasson College, Springvale, ME 04083

MR. VICTOR H. AULESTIA, Lang. Lab. Dir., U. of Maryland, 5401 Wilkins Ave., Baltimore, MD 21228

ROBERT P. AUSTIN, Lang. Lab. Dir., Loyola Academy, 1100 North Laramie, Wilmette, IL 60091

MS A. AYALA, Rollins College, Winter Park, FL 32789

\section{- B -}

DR. L. E. BAAK, Ch. of Dept. of Mod. Lang., Morningside College, Sioux City, IO 51106

J. W. BABIN, Dept. of French, St. F. X. Univ., Antigonish, Nova Scotia, Canada

MS MARY BAECHLE, Crystal Lake Community High School, 45 West Franklin St., Crystal Lake, IL 60014

REID E. BAKER, Ohio Dept. of Education, 781 Northwest Blvd., Columbus, OH 43212

ROBERT L. BAKER, Chairman Dept. of Russian, Middlebury College, Middlebury, VT 05753

MARIO BALBI, Benedictine Military School, 6502 Seawright Dr., Savannah, GA 31406

VIVIJA N. BALTINS, Loyola U. Library, 6525 N. Sheridan, Chicago, IL 60626

PAUL P. BALTIS, Lang. Lab. Supervisor, Leeward Community College, Pearl City, HI 96782

MS SHARON BARGER, Lang. Lab. Supervisor, Woodstock High School, Woodstock, IL 60098

CHARLES J. BARR, Lang. Lab. Dir., St. John's College, 5118 Seminary Rd., Camarillo, CA 93010

DR. RICHARD BARRUTIA, Universidad de California, Centro de Estudios en Mexico, Musee Nacional de Antropologia, Apart. Post. 6-884, Mexico $6 \mathrm{DF}$, Mexico

BARSTOW UNIFIED SCH. DIST,, 551 South 'H' St., Barstow, CA 92311

PROF. ERIC W. BAUER, 852 Erlangen, Hindenburgstrasse 52, West Germany MRS. FRANCES BECK, U. of Chicago, 5832 S. Stony Island, Chicago, IL 60637

MS BARBARA BEIMLY, Program Coordinator, Ohio State U. Listening Center, 155 Dieter Cunz Hall, Columbus, OH 43210 


\section{Membership Directory}

KEITH R. BELL, Dept. of Modern Lang., Mid-America Nazarene College, 2030 College Way, Olathe, KN 66061

MR. WILLIAM BEMERSDORFER, Ohio State U., Listening Center, Columbus, $\mathrm{OH} 43210$

JAMES BENNETT, PTY Ltd., 4 Cellaroy St., Collaroy, NSW Australia

FOR. LANG. DEPT., Bergen Comm. College, 400 Paramus Rd., Paramus, NJ 07652

FRERE ALPHONSE BERTRAND, La Bibliotheque, Ecole Normale N D de Foy, Cap Rouge, Quebec, Canada

MS ELIZABETH M. BISCHOFF, Dunbar HS, 307 Rowland Dr., Lynchburg, VA 24503

BISHOP HALL LANG. DEPT., Kaplama Heights, Honolulu, HI 96817. Att. Mr. Gemmer

CHARLES H. BLATCHFORD, 476 Kekupua St., Honolulu, HI 96825

PROF. V. BOBETSKY, Dir. of the Lang. Lab., Polytechnic Inst. of Brooklyn, 33 Jay St., Brooklyn, NY 11201

MS MARY BORRA, Supr. of For. Lang., Warwick Veteran's Memorial HS, 34 Warwick Lake Ave., Warwick, RI 02889

HERBERT BOSWAU, Box 8198 U.N.D. Station, Grand Forks, ND 58201

MR. ROGER BOURGARD, Dir. of the Lang. Lab., NCCU, Box 19371, Durham, NC 27705

DIR. LANG. LAB., Bowdoin College, Brunswick, ME 04011

LOUISE H. BOWEN, 201 Bartlett Hall, Dartmouth College, Hanover, NH 03755

LIBRARY, Bowie State College, Bowie, MD 20715

PATRICK C. BOYDEN, Dir. of the Lang. Lab., Kent State U., Kent, OH 44240

MR. ROBERT S. BRASHEAR, Assoc. Prin., Peters Township School District, 264 East McMurray Road, McMurray, PA 15317

MS GENEVIEVE BRASSIE, PO Box 293, Tiburon, CA 94920

SHERROD L. BRAXTON JR., Asst. Prof. of French, 7777 S. Lewis, Tulsa, OK 74136

MS BECKY A. BRIGGS, Michigan State U., PO Box 106688, Lang. Labs A126 Wells Hall, E. Lansing, MI 48823

BRIGHAM YOUNG U., Clark Library, Serials Section, Provo, UT 84601

MRS. F. C. BRITTAIN, West Chicago Community High School, 326 Joliet St., West Chicago, IL 60185

BRONX COMMUNITY COLLEGE LIBRARY, 120 East 184th St., Bronx, NY 10468

DR. LUTHER F. BROSSMAN, Albright College, Reading, PA 19604

BROWN UNIVERSITY, Box E, Lang. Lab. Library, Providence, RI 02912

C. P. BROWN, For. Lang. Dept., Western Kentucky U., Bowling Green, KY 42101

GEORGE BROWN COLLEGE, Hope Fairley - 37724, PO Box 1015, Station "B", Toronto, Ont., Canada

ROBERT BROWN, Dir. of the Lang. Lab., 1100 Harvard St., Washington, DC 20009 
PROF. WILBUR J. BRUNER, William Jewell College, Liberty, MO 64068 MRS. JUNE BRUST, Upper Moreland Senior HS, Terwood Rd., Willow Grove, PA 19090

SR. M. ALBAN BSHARAH, Dir. Lang. Lab., College of New Rochelle, New Rochelle, NY 10801

DR. GEORGE C. BUCK, Lang. Lab. Dir., U. of Washington, 118 Denny Hall, Seattle, WA 98105

YOLANDA BURR, R D 3, Wellsville, NY 14895

ANGEL M. BUSTELO, Instructional Services Center, Rm 312, 707 E. Columbus Dr., Tampa, FL 33601

E. H. BUTLER LIBRARY, Box P, State University College, 1300 Elmwood Ave., Buffalo, NY 14222

BUTTE JUNIOR COLLEGE, P O Box 566, Durham, CA 95938, Attn. Richard A. Clark

E. BUTTERWORTH, Asst. Language Training Advisor, Citizenship Branch, 20 Spadina Road, Toronto, Ontario, Canada

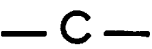

LIBRARY, California State Polytech College, Periodicals Section, San Luis Obispo, CA 93401

DR. CALLAHAN, 820 North Michigan Ave., Chicago, IL 60611

CALVIN LIBRARY, 3207 Burton SE, Grand Rapids, MI 49506

MRS. G. M. CAMPBELL-Librarian, Senior HS Library, 160 Broad St., Bloomfield, NJ 07003

ROBERT W. CANNADAY, JR., Foreign Lang. Section, P O Box 2360, Honolulu, HI 96804

PIERRE J. CAPRETZ, Dir. of the Lang. Lab., Yale University, 111 Grove Street, New Haven, CT 06510

FLORENCE CARLSON, Dir. Audiovisual Services \& Lang. Lab., Wellesley College, Wellesley, MA 02181

MR. MAX CARMICHAEL, Washington Twp. Pub. Schools, 1801 East 86th St., Indianapolis, IN 46240

LIBRARY, Carson-Newman College, Jefferson City, TN 37760

KENNETH CARTER, Long Beach City College, For. Lang. Dept., 701 Locust Ave., Long Beach, CA 90813

F. A. CARTIER, 1029 Forest Ave., Pacific Grove, CA 93950

CENTRE FOR APPLIED LINGUISTICS, Popik Bldg., Hebrew University, Jerusalem, Israel

CENTRE FOR INFO ON LANG. TEACHING, Deputy Librarian, State House, High Holborn, London, WC 1, England

MS CHRISTINE E. CERVIZZI, Coordinator of For. Lang., Northbridge HS, Linwood Ave., Whitinsville, MA 01588

CHAFFEY UNION HIGH SCHOOL, 211 Fifth St., Ontario, CA 91762

DEPT. OF FOR. LANG., Chaffey Union HS Dist., 211 W. 5th St., Ontario, CA 91762

GERALD K. J. CHANG, 4457 Puu Panini Ave., Honolulu, HI 96816

MS ROSALIE M. CHEATHAM, Lang. Lab. Dir., U of Arkansas, 6405 Evergreen Rd., Little Rock, AR 72207 


\section{Membership Directory}

CHARLES MAURICE CHERRY, Apt. 416 Englehart Hall, 1915 Maple Ave., Evanston, IL 60201

MISS TOSHIKO CHOMEI, Instructor, Ochanomizu University, Otsuka 2 Chome, Bunkyo-ky, Tokyo, Japan

HENRY R. CHRISTMAN, Dir. of Lang. Lab., Kutztown State College, 215 Wellington Ave., Lincoln Park, Reading, PA 19609

WILLIAM CLAPPER, MFL Consultant, Missouri State Dept. of Educ., P O Box 480, Jefferson City, MO 65101

CLARK U., Dept. of Romance Lang., Worcester, MA 01610

CALVIN A. CLAUDEL, Prof. of French \& Spanish, Box 622, Salisbury State College, Salisbury, MD 21801

THELMA CLINE, Dir. of the Lang. Lab., Adelphi University, Garden City, NY 11530

RALPH S. COLLINS, Dept. of For. Lang., Maryville College, 1741 Linda La., Maryville, TN 37801

COMM. AND ARTS DIV - CCD, North Campus, 1001 East 62nd, Denver, CO 80216

COMMUNITY COLLEGE OF PHILADELPHIA, Library, 34 South 11th St., Philadelphia, PA 19107

PAUL R. CONNORS, For. Lang. Coordinator, 37 Pine Grove Ave., Sharon, MA 02067

DR. MARILYN J. CONWELL, 909 Concord Bldg., Valley View Apts., 251 W. DeKalb Pike, King of Prussia, PA 19406

c. T. COOPER, Dept. of Spanish, Lebanon Valley College, Annville, PA 17003

HENRY F. COOPER, Lang. Lab. Dir., U of Oregon, Eugene, OR 97403

M. GERARD COUROUX, College Marie-Victorin, 7000 Rue Marie-Victorin, Montreal, 462, Quebec, Canada

J. L. COVA, Dept. of French, Queen University, Kingston, Ontario, Canada

SHIRLEY O. COX, Auburn U., For. Lang. Dept., Auburn, AL 38630

JOHN F. CREAMER, Dir. of the Lang. Lab., Hudson HS, Cottage St, Hudson, MA 01749

PROF. JOHN E. CREAN, JR., Dept. of European Lang., U of Hawaii - Moore Hall, Honolulu, HI 96822

LEONARD R. CRIMINALE, Dir. of the Lang. Lab., Elmira College, Elmira, NY 14901

MS LAUREL CROOKS, Dir. Lang. Lab., Northwestern U., Rm 48B Kresge Cent. Hall, Evanston, IL 60201

DR. DAVID CROWNER, Dir. of the Lang. Lab., Gettysburg College, Gettysburg, PA 17325

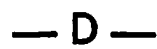

BROTHER EDWARD DAILEY, Chm of the Lang. Dept., St. Edward HS, 13500 Detroit Ave., Lakewood, OH 44107

SUPT. OF SCHOOLS, PO Box 1167, Darien, CT 06820

MICHEL L. DARRAS, Deputy Dir. of School Translators, Laurentian U., Sudbury, Ontario, Canada 
WALTER F. DAVISON, Director of the Lang. Lab., Old Dental Clinic, U of Pittsburgh, Pittsburgh, PA 15213

PHILIP S. DAY, Dept. of French, Queen's U, Kingston, Ont., Canada

UNIVERSITY OF DAYTON LIBRARY, Periodical Dept., U of Dayton, Dayton, $\mathrm{OH} 45409$

DR. G. DE BONE, Head of the Lang. Dept., LaGrange College, LaGrange, GA 30240

MS X. de GERY, Crystal Springs School for Girls, 400 Uplands Dr., Hillsborough, CA 94010

DE LA SALLE COLLEGE LIBRARY, PO Box 3819, 2401 Taft Ave., Manila, Phillipines

MR. FRANCIS V. DELLY, Dept. of For. Lang., Staten Island Community College, 715 Ocean Terrace, New York, NY 10301

MR. D. DERRY, Director of the Lang. Lab., Waterloo University, Waterloo, Ontario, Canada

BENNIE T. DE SALVO, Hall High School, 50 South Main Street, West Hartford, CT 06051

DANIEL DETI, Lang. Dept., College of the Desert, Palm Desert, CA 92260

EMILIO E. DE TORRE, Queens College of the City U of NY, Flushing, NY 11367

WILLIAM C. DICKERMAN, Lang. Lab. Dir., U of Houston, 3801 Cullen Blvd., Houston, TX 77004

VITO J. DI VINCENZO, Director of the Lang. Lab., Villanova University, Spring \& Hilldale Road, Malvern RD 2, PA 19355

DR. O. DRAGONE, Dir. MLS, AIC, State St., Springfield, MA 01109

AGNES DUNAWAY, For. Lang. Dept., Riverside HS, 1615 E. Locust St., Milwaukee, WI 53211

MR. BILL DURHAM, Clemson U., 201 Strode Tower, Clemson, SC 29631

DURHAM TECH. INSTITUTE, Att.: Library, 1637 Lawson St., Durham, NC 27703

F. A. DU VAL, Dept. of Class. and Mod. Lang., Cornell College, 710 8th Ave. N., Mt. Vernon, IO 52314

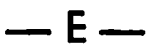

EASTERN MONTANA COLLEGE LIBRARY, Serials Dept, Billings, MT 59101

GEORGE M. EBERHARDT, Tech. Dir., Lang. Lab., Drew U, BH 217, Madison, NJ 07940

R. M. ECONOMOS, Pace College, 41 Park Row, New York, NY 10038

WALTER EGGMAN, Dept. of For. Lang. - Boys Div., Bishop Reilly Diocesan HS, F. Lewis \& LI Expressway, Fresh Meadows, NY 11365

DR. T. L. EICHMAN, Asst. Prof. of German, Indiana State U., 8600 University Blvd., Evansville, IN 47712

JAMES D. EK, Dir. of the Lang. Lab., Western Michigan U., Kalamazoo, MI 49001

RICHARD H. ELDRIDGE, Inst. of Spanish, PO Box 46, West Va. Wesleyan College, Buckhannon, WV 26201 


\section{Membership Directory}

RAY ELLIS, Hardin-Simmons U., Abilene, TX 79601

WILLIAM E. ELMENDORF, Lang. Lab. Dir., Western Washington State Col., Bellingham, WA 98225

ALBERT EMANUEL LIBRARY, U. of Dayton, Dayton, OH 45409

EDMOND L. EMERY, Foreign Lang. Dept., Milford Academy, 150 Gulf St., Milford, CT 06460

THERESE EMILIENNE, Dir. of the Lang. Lab., Annhurst College, Woodstock, CT 06281

ENGLISH LANG. SCHOOL, DLIELLS, Building 7514, Lackland Air Force Base, TX 78236

ERIK ERAMETSA, Language Laboratory, University of Jyvaskyla, Finland

C. T. ERICKSON, Prof.-Staten Island Com. College, 176 Arlo Rd., Staten Island, NY 10301

EXPERIMENT IN INTERNATIONAL LIVING, Putney, VT 05346

\section{$-\mathbf{F}$}

JAMES C. FAULKNER, State University College, 17 Cedar Ridge Road, New Paltz, NY 12561

LEO J. FECTEAU, Hd. of the For. Lang. Dept., Barrington Pub. Schools \& HS, Barington, RI 02806

FELSOOLTATASI PEDAGOGIAI, High School Research Centre, Kutatolozpont VIII, Rigo u. 16, 1389 Budapest 62, P O B 149, Hungary

DAVID FERNANDEZ, Dept. Lang. Lit. \& Ling., Tillett Rm 301, Livingston College, New Brunswick, NJ 08900

MRS. FERRIS, Audio-Visual Dept., Fulton-Montgomery Comm. College, RR 67, Johnstown, NY 12095

A. V. FICARRA, Lang. Dept., Western Connecticut St. College, 181 White St., Danbury, CT 06810

BARTHOLOMEW J. FINN, Dir. of Lang. Lab., Skill Center, 1485 Market St., San Francisco, CA 94103

FISHER LIBRARY, Serials Dept. 134881, University of Sydney, Sydney, New South Wales, Austraila 2006

BRUCE C. FITCH, Dir. Lang. Lab., Transylvania College, 300 N. Broadway, Lexington, KY 40508

RALPH FITTANTE, Tashua School, 401 Stonehouse Rd., Trumbull CT 06611

DONALD N. FLEMMING, For. Lang. Dept., Keene State College, 17 Gardner St., Keene, NH 03431

LIBRARY, Florida State U., Tallahassee, FL 32306

PROF. P. FRED FLUCKIGER, Direktor Abteilung fur angewandte, Linquis. tick der Universitat Berne, Langgasse 7, 3012 Berne, Switzerland

ROBERT FOURNIER, For. Lang. Consultant, State Dept. of Education, 17 Webster St., Suncook, NH 03275

SIMON FRASER U. LIBRARY, Acquisition Division-Serials, Burnaby 2, BC, Canada

ERNEST A. A. FRECHETTE, Dept. of Mod. Lang., Florida State U., Talla hassee, FL 32306

FREEHOLD HIGH, Att.: For. Jang. Dept., Robertsville Rd., Freehold, NJ 07728 
MS MARGARET Z. FREEMAN, Dir. Lang. Lab., Mass. Inst. of Technology, Room 14N 222, Cambridge, MA 02139

FRANK M. FRIEDMAN, Dir. of Lang. Lab., Flint Community Jr. College, 1401 E. Court St., Flint, MI 48503

HANS J. FRITSCH, PO Box 61, Whately, MA 01093

ROBERT KARL FRITZ, A/V Coord. for the Dept. of For. Lang., Ball State U., 1353 East Jackson St., Muncie, IN 47305

ARTHUR F. FROST, Dir. of Lang. Lab., Princeton U., 304 E. Pyne Bldg., Princeton, NJ 08540

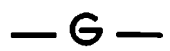

ALAN GARFINKEL, Purdue U., Dept. of Mod. Lang., Lafayette, IN 47907

ROGER T. GAUTIER, Dir. of the Lang. Lab., U. of Saskatchewan, Saskatoon, Saskatchewan, Canada

GENO R GEMMATO, Audio Lab-Eaton Hall-122, Tufts U., Medford, MA 02155

N. GENDREAU, Mod. Lang. Dept., Rhode Island College, 600 Mount Pleasant Ave., Providence, RI 02908

LIBRARY, Genesee Community College, 3837 W. Main St., Batavia, NY 14020

MRS. M. G. GENNAULA, 5872 Kings School Road, Bethel Park, PA 15102

SR. JUDITH GEORGE, College of Great Falls, 1301 20th St. South, Great Falls, MT 59401

GERMAN/CLASSICS/RUSSIAN DEPT., Sacramento State College, $6000 \mathrm{~J}$ St. UPO\# 1933, Sacramento, CA 95819

GERMAN CENTER BOSTON, Dir. of the Lang. Div., 170 Beacon St., Boston, MA 02116

MIGUEL ANGEL GIELlA, Carleton U., Spanish Dept., Colonel By Drive, Ottawa, Ontario, Canada

JYOSHI TANKI GIFU, Daigaki Toorin Nagara, Gifu 500, Japan

S. ROSENDA GILL, Lang. Lab. Coordinator, Regis Col., Weston, MA 02193

GILMAN SCHOOL, Lang. Lab., 5407 Roland Ave., Baltimore, MD 21210

ARTHUR J. GIONET, Dir. of the Lang. Lab., North Texas State U., Denton, TX 76203

E. M. GOLDSTEIN, Faculty of Education, U. of Ottawa, Ottawa 2, Ontario, Canada

T. R. GOLDSWORTHY, Dir. Lab. for Rec. Instr., U. of Wisconsin, Madison, WI 53706

ALBERT A. GOODRICH, Lang. Dept., Joint School District 1, Three Lakes, WI 54562

ALAN M. GORDON, Dir. of the Lang. Lab., U. of Toronto, Dept. of Ital. \& Hispania Studies, Toronto 5, Ontario, Canada

PETER B. GORE, Dir. of the Lang. Lab., Deerfield Academy, Deerfield, MA 01342

NOELLA GOULET, Dept. of French, Notre Dame College, 2321 Elm St., Manchester, NH 03104

MS RUTH L. GREENE, Dir. of Lang. Lab., The Karl E. Weston Lang. Center, Williams College, Williamstown, MA 01267 
CLARA GREGORY, Spanish Consultant, Texas Education Agency, 201 East 11th St., Austin, TX 78701

JOSEPH F. GREIPP, 119 Sherwood Drive, Greenwood Park, Blackwood, NJ 08012

FRANK GRITTNER, 710 Woodward Dr., Madison, WI 53704

GLEN GROSJEAN, Lang. Lab. Asst. Dir., U. of California, B-40 Dwinelle Hall, Berkeley, CA 94720

STEPHANIA E. GROSS, Bowling Green State U., University Hall, Bowling Green, OH 43402

ALLAN W. GRUNDSTROM, Dept. of French, Bucknell U., Lewisburg, PA 17837

V. A. GUADINO, Modern Lang. Center, 252 Bloor Street West, Toronto 181, Ontario, Canada

MR. PAUL GUENETTE, Dir. Lang. Lab., Brookline HS, 115 Greenough St., Brookline, MA 02146

SISTER M. GEORGE GUERTIN, Mt. St. Mary College, Hooksett, NH 03106

EDWARD M. GUGGER, For. Lang. Dept., Medford HS, Medford, MA 02155

DR. JEAN GUITTON, Georgia College, Milledgeville, GA 31061

MARJORIE GUSTAFSON, Lang. Lab. Dir., Macmurray College, 1419 Lakelawn Drive, South Jacksonville, IL 62650

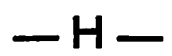

MRS. DONALD F. HAGEN, Dir. of Lang. Lab., Fairleigh Dickinson U., Florham Madison Campus, 285 Madison Ave., Madison, NJ 07940

HALTON COUNTY BOARD OF EDUCATION, Box 524, 2050 Guelph Line, Burlington, Ontario, Canada, Attn.: M. M. Robinson \& Mr. Thatcher

D. HAMMOND, Spanish Lang. Pub. Service, 211 South Main St., McAllen, TX 78501

ERWIN M. HAMSON, U. of Michigan, Dir. of the Lang. Lab., 1401 Mason Hall, Ann Arbor, MI 48104

DAVID A. HANSON, Russian Dept., Brandeis U., Waltham, MA 02154

GLENN HAREWOOD, Humber College of Appl. Arts \& Tech., Humber College Blvd., Rexdale, Ontario, Canada

G. CHRISTOPHER HARTLEY, Head of the Lang. Dept., The Peddie School, Hightstown, NJ 08520

V. HAUGHTON, French Dept., MacDuffie School, Springfield, MA 01105

DR. MAURICE M. HEIDINGER, For. Lang. Dept., Eastern Montana College, Billings, MT 59101

HENDERSON COMMUNITY COLLEGE, Lang. Lab., Henderson, KY 42420 ROBERT L. HENDRICKSON, 925 Nooney St., Poplar Bluff, MO 63901

ROBERT HENNEBERGER, Dir. of the Lang. Lab., U. of Florida, Gainesville, FL 32601

MS DOROTHY B. HENRY, 131 Terrace Rd., Levittown, PA 19056

- ADELE B. HERNANDEZ, Spanish Dept., U. of Maine, 96 Falmouth St., Portland, ME 04103 
ROBERT F. HERON, For. Lang. Dept., Bellingham HS, Bellingham, MA 02019

LAZAR OM. HERRERA, Dir. of the Lang. Lab., The Westminster Schools, 1424 W. Paces Ferry Rd. NW, Atlanta, GA 30327

DR. J. HEWSON, Memorial University of Newfoundland, St. John's, Newfoundland, Canada

MRS. MARGARET HILTS, Loma Linda U., Riverside, CA 92505

MARGIE HINKLEY, Coordinator Lang. Lab., Inter American U., PO Box 1293, Hato Rey, Puerto Rico 00919

HIROSHIMA DAI, Honbu Toshokan-k, Hiroshima, 730 MZ Japan

BERNICE G. HIRSCH, Dir. of the Lang. Lab., Samford U., 800 Lakeshore Dr., Birmingham, AL 35209

ALBERT M. HOOD, For. Lang. Dept., Eastbrook HS, RR 6, Marion, IN 46952

WM. HORSTMAN, Mitchell Hall 104, Dir. Lang. Lab., U. of Wisconsin-Milwaukee, WI 53211

HOWELL HS, Squankum Yellowbrook Rd., Farmingdale, NJ 07727, Attn.: Media Center

ROBERT T. HOWLING, English Dept., New Haven College, New Haven, CT 06505

DONALD T. HUFFMAN, For. Lang. Dept., West Virginia U., 781 Meadowbrook Rd., Morgantown, WVA 26505

DR. JOSEPH C. HUTCHINSON, Academic Advisor, HQ DL 1, Washington Navy Yard (AA), Washington, DC 20390

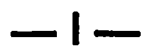

D. A. IANNUZZI, I' ’pt. of Mod. Lang., Niagara University, Niagara University, NY 14109

I M C, 201 E. Nine Mile Rd., Highland Springs, VA 23075

INST. f DOLMETSCHER AUSBILDUNG, Univ. Innsbruck, Innsbruck 6020, Austria

INSTITUTE DE LING APPL., Fac des Langes, Litt et Civ Etrange, Universite des Sciences Humaines, 25 Rue de Soleil, 67 Strasbourg, France

INTER AMERICAN $\mathcal{C}$. of PUERTO RICO, Library, San German, Puerto Rico 00753, Attn.: Mr. Hsu.

DR. EVELYN U. IRVING, Maryville College, 1840 Azrock Dr., Knoxville, TN 37914

IRWIN LIBRARY, Butler University, Indianapolis, IN 46208

PROF. MICHAEL ISSACHAROFF, Dept. of French, U. of Western Ontario, London 72, Ontario, Canada

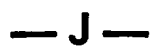

GORDON JACKSON, Dir. of the Lang. Lab., Capital U., 2199 East Main, Columbus, OH 43209

FERNAND JACQUES, For. Lang. Dept., Magnificat HS, 13429 Everson Ave., Lakewood, OH 44107 


\section{Membership Directory}

MR. H. W. JAMIESON, 22 Thornlee Cr., N W, Calgary 47, Alberta, Canada

JOSEPHY J. JANES, Supr. of Pub. Instruction, Acad. Affairs-For. Lang. Unit, 316 S. Second St., Springfield, IL 62706

FRANCIS G. JARLETT, Dir. of the Lang. Lab., Central Conn. State College, Depart. of Mod. Languages, New Britain, CT 06050

JEFFERSON STATE JR. COLLEGE, Dept. of For. Lang., Attn.: Martin O'Rourke, Bethune Deramus 241, Birmingham, AL 35215

MR. JOHANNES JESPERSON, Mod. Lang. Dept. Rhode Island Jr. College, 199 Promenade St., Providence, RI 02908

ALAN JOHNS, Electronics Tech., University of Windsor, Sunset Avenue, Windsor II, Ontario, Canada

MS BARBARA JOHNSON, Washington State U., For. Lang. Dept., Pullman, WA 99163

DR. ROBERTA JOHNSON, Dir. Lang. Lab., Claremont Men's College, Claremont, CA 91711

R. J. JONES, College of Education, University of Toronto, 371 Bloor Street West, Toronto, Ontario, Canada

DENIS JUHEL, Dept. of Romance Lang., U. of New Brunswick, Fredericton, New Brunswick, Canada

JOAN N. JUKICH, Lang. Lab. Dir., U. of Minn., Duluth, 428 N. 41 Ave. E.. Duluth, MI 55804

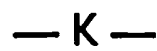

DR. KADAR, Napa College, 2277 Napa-Vallejo Hwy., Napa, CA 94558

KAMEHAMEHA SCHOOLS, Bus. \& Finance Dept., Administration Bldg., Honolulu, HI 96817

EDMUND J. KAMINSKI, Dept. of Mod. Lang., Marietta College, Marietta, OH 45750

MRS. F. C. KATAY, Lang. Lab. Dir., 1019 1st St. NW, Calgary, Alberta T2M2S2, Canada

KAUPPAKORKEAKOULU, Runeberginkatu 14-16, Helsinki 10, Finland

-yUSUKE KAWARABAYASHI, 145 Bernice Ave., U. of the Pacific, Stockton, CA 95207

MS P. KEENAN, 2682 17th Ave., San Francisco, CA 94116

MS CECI KELLINGER, Lab. Director, Wilson College, Chambersburg, PA 17201

SISTER BETH KELSO, Dir. of the Lang. Lab., St. Mary of the Woods College, St. Mary of the Woods, IN 47876

JOSEPHY KEMPRECOS, Dir. of the Lang. Lab., Rumson-Fair Haven Regional HS, Rumson, NJ 07760

MS DAPHENE KENNEDY, Dept. of Mod. Lang., Freed-Hardeman College, Henderson, TN 38240

MS DORA F. KENNEDY, Supr. of For. Lang., Prince George's Public Schools, 4806 Harvard Rd., College Park, MD 20740

LANG. LAB, U. of Kentucky, 312 Classroom Bld., Lexington, KY 40506 
MR. GLEN KERCH-Dir., Asociados Linguisticos, Apartado Postal \# 2562, Managua, Nicaragua

MRS. CHARLOTTE W. KERR, Lang. Lab. Supervisor, Occidental College, 1600 Campus Rd., Los Angeles, CA 90041

DR. ARTHUR S. KIMMEL, For. Lang. Dept., Western Washington State Col., Bellingham, WA 98225

KINDERMANN LTD., 3300 Cavendish Blvd., Suite 100, Montreal 261 P Q, Canada

SERIALS LIBRARIAN, Paul Klapper Library, Queens College, Flushing, NY 11367

WALLACE G. KLEIN, Business Mgr., The Modern Language Journal, 13149 Cannes Drive, St. Louis, MO 63141

SHIRLEY KOENEN, 2420 Appleside Blvd., Clarkston, WA 99403

EGYETEMI KONYVTAR, P O Box 483, Budapest 5, People's Republic of Hungary

COMMANDANT G. KOZORIZ, Canadian Forces FLS, Dept. of National Defense, Ottawa 4, Ontario, Canada

MRS. KRANE, Scotch Plains Sr. HS, Westfield Rd., Scotch Plains, NJ 07076 EDWARD L. KRUSE, JR., Lecturer in Education, Canisius College, Buffalo, NY 14208

ROY L. KRYNITZ, Lang. Lab. Dir., New Mexico State U., University Park, NM 88001

SHOICHI KURIYAMA, Waseda Univ., 3-12-16 Shimoigusa Suginami, 167 Tokyo, Japan

JOHN F. KUNKLE, Lang. Lab., U. of Southwestern Louisiana, Lafayette, LA 70506

\section{$-\mathrm{L}-$}

MR. ARTHUR A. LEONE, 2593 Shell Drive, Indiana, PA 15701

LETHBRIDGE U. LIBRARY, Acquisitions Div., Lethbridge, Alberta, Canada

MRS. B. LEVINSON, Brooklyn College-City U., Supr. Lang. Lab., Bedford Ave. and Ave. H, Brooklyn, NY 11210

MARCEL LIDJI, Lecturer CCNY, Romona Lang. Lab., 133 and Convent Ave., New York, NY 10031

DAVID W. LIGHTCAP, Audio Visual Dir., Susquehanna U., Selinsgrove, PA 17870

DR. TORSTEN LINDBLAD, Kortvagsgatan 14, S-421 33 Vastra Frolunda, Sweden

LUIS LINDNER, Lang. Lab. Dir., Stephens College, Columbia, MO 65203 LINGUAPHONE INSTITUTE LTD., 207-209 Regent St., London, England

PROF. WM. A. LITTLE-Editor, German Quarterly, Dept. of Germanic and Slavic Lang., U. of Virginia, Charlottesville, VA 22901

SISTER ELAINE LOUBERT, Chairman, College of St. Scholastica, Duluth, MI 55811

PAUL LUCKAU, Brigham Young U., 215 McKay Building, Provo, UT 84601 RONNI LACROUTE, Suffolk U., 41 Temple St., Boston, MA 02114 
RAYMOND J. LADINSKY, JR., Lang. Lab. Dir., New Trier HS East, Winnetka, IL 60093

RAYMOND J. LAGUEUX, Acting Chmn., Dept. of For. Lang., U. of Maine at Farmington, Farmington, ME 04938

DALE V. LALLY, JR., Dir. Lang. Labs., Marquette U., Milwaukee, WI 53233

DR. RAYMOND LAMFRAND, Head Programmed French Courses, Centre Linguistique d'Ottawa, Chemin Carson 800 A225, Ottawa K1A0M7 Canada

DALE L. LANGE Prof., Univ, of Minnesota, 148 A Peik Hall, Minneapolis, MI 55455

THE LANGUAGE CENTRE, Arts South, Univ. of Melbourne, Parkville, Victoria 3052, Australia

LAPPEENRANNAN TEKNILLISEN KORKEAKOULUN, Kirjasto, Taipalsaarentie, 53900 Lappeenranta 90, Finland

SIR WILFRID LAURIER COLLEGE, 145 Guildwood Parkway, Moderns Dept., Scarboro, Ontario, Canada

MISS KAREN LAWRENCE, Park Jr. HS, Scotch Plains, NJ 07076

AMADO M. LAY, Asst. Prof. of Spanish, Gustavus Adolphus College, Box 1413, St. Peter, MI 56082

BUCHHANDLUNG LEHMKUKL, 8000 Munchen 23, Leopoldstrasse 45, Fed. Republic of Germany

\section{$-\mathrm{M}-$}

FWELIS MAC EINRI, Director Language Centre, Colaiste San Froinsias, Germanton Co. Na. Mi, Ireland

JOHN MACINKO, Lang. Lab. Dir., Old Main Bldg., U. of Colorado, Boulder, CO 80302

LOLA A. MACKEY, 4848 North Woodmere, Fairway No. 7, Scottsdale, AZ 85251

W. W. MACKIEWICZ, Lecturer, Institut fur Anglistik und Amerikanistik, Technische Universitat, 33 Braunschweig, Wendenring 1, Fed. Rep. of Germany

DR. ROBERT MAGILL, Dir. Lang. Lab., Towson State College, Dept. Mod. Lang., Baltimore, MD 21204

JOHN C. MANOLIS, Lang. Lab. Dir., Mary Washington College, PO Box 1126 College Sta., Fredericksburg, VA 22401

SR. M. V. MAPA, Lang. Lab. Dir., U. of San Diego, Alcala Park, San Diego, CA 92110

SR. MARIE THERESE, Chrm. For. Lang. Dept., College of White Plains, 52 North Broadway, White Plains, NY 10603

MARQUETTE U., Dept. of For. Langs. \& Lits., 526 N. 14th St., Milwaukee, WI 53233

D. W. MARSHAIL, Vancouver City College (Langara), $100 \mathrm{~W}$ 49th Avenue, Vancouver, BC Canada

EDWARD MARXHEIMER, Lang. Lab. Dir., U. of Alberta, Edmonton, Alberta, Canada

D. ARTHUR W. McCARDLE, Dir. Lang. Lab., Gettysburg College, German Dept., Gettysburg, PA 17325 
LIBRARY, C. L. McClatchy Sr. HS, 3066 Freeport Blvd., Sacramento, CA 95818

PROF. J. T. McCULLOUGH, Lang. Lab. Dir., Dept. of Spanish and Portuguese, California State U., Sacramento, CA 95819

JERRY McCUNE, Dir. Lang. Labs., 120 Ballantine Hall, Indiana U., Bloomington, IN 47401

MS HELEN McGETTRICK, 115 St. Stephen St., Boston, MA 02115

McGILL U. LIBRARY, Serials Dept., 3459 McTavish St., Montreal, 112 Quebec, Canada

DEPT. OF FOR. LANGS., Memphis State U., Memphis, TN 38152

MERCED COLLEGE, 3600 M St., Merced, CA 95340

C. A. MESSNER, Assoc. Prof. of Mod. Lang., Carleton College, Northfield, MI 55057

JOHN METCALF, 29 Willow Rd., Menlo Park, CA 94025

MICHIGAN U. LIBRARY, Lang. \& Lang. Behavior Abstracts, 220 East Huron St., Ann Arbor, MI 48103

MICHAEL MICKO, Lang. Lab. Dir., Widener College, Chester, PA 19013

ARTHUR L. MICOZZI, Supr. of For. Lang., Board of Educ., Baltimore Co., 2401 Lamport La., Baltimore MD 21234

MRS. MARTHA R. MLLER, Admin. Asst.Harvard U., Mod. Lang. Center, Boylston Hall G-3, Cambridge, MA 02138

ROBERT C. MILLER, Chmn. Modern Lang. Dept., The Thacher School, Ojai, CA 93023

SHELBY C. MILLER, Dir., Geddes Lang. Center, Boston U., Rm. 540, 685 Commonwealth Ave., Boston, MA 02215

DR. DOROTHY HURST MILLS, Chmn. Dept. of For. Lang., Chapman College, Orange, CA 92666

U. OF MINNESOTA, DULUTH, Lang. Dept. H455, Duluth, MI 55812

DEPT. OF FOR. LANG., U. of Missouri, Rm. 409 Haag Hall Annex, Kansas City, MO 64110

MIRCO M. MITROVICH, PH D., Asst. Prof., Sunrise Acres Rt. 1, New Concord, OH 43762

MARLIN M. MITTAG, Lang. Lab. Dir., East High School, 710 Garfield Ave., Aurora, IL 60506

MS MARY E. MOEN, Instructor, Anoka Ramsey State Jr. College, 7154 NE Riverview Terrace, Minneapolis, MI 55432

THE LIBRARIAN, Monash Teacher's College, Blackburn Rd., Clayton, Victoria 3168, Australia

MONASH UNIVERSITY LIBRARY, Periodicals Dept., Clayton, Victoria 3168, Australia

FRANCES MONGILLO, 179 Hunting Hill Ave., Middletown, CT 06457

DIRECTEUR SERVICES AUXILIAIRES, Montreal Catholic HS Commission, Adult Education, 3737 East Sherbrooke St., Montreal 406, Quebec, Canada

DR. MICHAEL J. MOORE, Mesa College, San Diego, CA 92111 
MOORHEAD STATE COLLEGE, Library-Serials Dept., Moorhead, MI 56560

SISTER M. CATHERINE MORAN OP, Ch., Dept. For. Lang., Edgewood College of the Sacred Heart, 855 Woodrow St., Madison, WI 53711

VAL GUY MOREAU, Chmn., Mod. Lang. Dept., Portsmouth HS, Education La., Portsmouth, RI 02871

MR. JOSEPH F. MORRISON, Audio Information Service, U. of Illinois at Chicago Circle, Box 4348, Chicago, IL 60680

ROBERT R. MORRISON, Chmn. Mod. Lang., Southern Missionary College, PO Box 475, Collegedale, TN 37315

WORTH S. MOSER, Chm. Dept. of For. Lang., Darlington School, Rome, GA 30161

STEPHEN MOSS, A.V Dept., Regis College, West 50th and Lowell Blvd., Denver, CO 80221

CLIFF MOUNTAIN, Foreign Lang. Dir., Mesa College, Grand Junction, CO 81501

DOROTHY H. MOYNIHAN, Dir. For. Lang., Hamden HS, 2040 Dixwell Ave., Hamden, CT 06514

CHARLES V. MULHOLLAND, Lang. Lab. Supr., U. of Rhode Island, Dept. of Lang., Kingston, RI 02881

HAROLD T. MURPHY, Asst. Prof. in Span., Marshall U., Box 238, 16th and 3rd St., Huntington, WV 25701

THOMAS M. MYERS, Lang. Lab. Dir., Colorado State U., Fort Collins, Co 80521

\section{$-\mathrm{N}-$}

NANYANG UNIVERSITY, The Librarian, Upper Jurong Road, Singapore 22, Rupublic of Singapore

DONALD NASH, Instr. in French, Roberts Wesleyan College, Rochester, NY 14624

NASSAU COMMUNITY COLLEGE, Periodicals Unit, Stewart Ave., Garden City, NY 11530

NAT'L LENDING LIBRARY FOR SCIENCE \& TECHNOLOGY, Accessions Dept., Boston Spa, Yorkshire, England LS23 7BQ

NAT'L LIBRARY OF AUSTRALIA, Librarian, Preparation Branch (NS110/ 72), Canberra, ACT 2600, Austraila

CARROLL E. NAVES, Dir. Lang. Lab., Barry College, 11300 NE 2nd Ave., Miami Shores, FL 33161

ORRIN NEARHOOF, Acting For. Lang. Consult., Iowa Dept. of Pub. Inst., 2745 Bennett Ave., Des Moines, IA 50310

U. OF NEBRASKA AT OMAHA, Periodical Dept., Gene Eppley Library, Box 688, Downtown Station, Omaha, NB 68101

U. OF NEVADA, Library - Periodicals, Las Vegas, NV 89109

JAMES L. NEWMAN, Simmons College, 300 The Fenway, Boston, MA 02115

DR. LEONARD NEWMARK, Dept. of Ling., U. of California, La Jolla, CA 92037 
NEW YORK STATE U., Library SRLS Section, Vestal Pkwy. East, Binghamton, NY 13901

NORTH EAST LONDON POLYTECHNIC, Dept. of Arts \& Modern Lang.Longbridge Road, Dagenham Essex, RMB 2AS, England

NORTH TEXAS STATE U., Acquisitions Librarian, Box 5138, NT Station, Denton, TX 76203

WITOLD NOVAK, Lang. Lab. Dir., A-V Center, Monmouth College, Monmouth, IL 61462

J. STANLEY NUFFER, P O Box 751, Portland State U., Portland, OR 97207

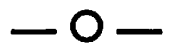

LANG LAB., Odense University, c/o OU Library, Periodicals Dept., Islandsgade 2, DK-5000 Odense, Denmark

DIR. OF MODERN LANG. LAB., Oklahoma Baptist U., Shawnee, OK 74801

MARCUS D. O'NEAL, JR., 134 Epler Dr., San Antonio, TX 78228

ONONDAGA COMMUNITY COLLEGE, Mrs. B. G. Lytle, Room 449, 700 E. Walter St., Syracuse, NY 13210

ONT. INST, STUD. EDUC., Library Periodicals, 252 Bloor St. West, Toronto 5, Ontario, Canada

ONT. INST: FOR STUDIES IN EDUC., Mod. Lang. Center, 252 Bloor St. West, Toronto, Ontario, Canada

OPPENHEIM, HERMINGHAUSEN \& CLARK, 3400 South Dixie, Dayton, OH 45439

MRS. IDA MARIE OWENS, For. Lang. Dept., Ramapo HS, George St., Frarklin Lakes, NJ 07417

MR. WILLIAM M. PARK, Dept. of For. Lang., UNC Charlotte Station, Charlotte, NC 28213

PARKLAND COLLEGE LIBRARY, 2 Main St., Champlain, IL 61820

DR. MILTON I. PATRIE, Dir. AV/TV Center, U. of Louisville, Louisville, KY 40208

GLORIA A. PATRON, Lang. Lab. Dir., Louisiana State U., Lake Front, New Orleans, LA 70122

MRS. JACQUELINE M. PAUCHET, Dir. Lang. Lab., Knoxville College, Knoxville, TN 37914

MS CHRISTA E. PAUL, Dir. Lang. Lab., Instr. of German, Phila. College of Pharmacy \& Sci., 43rd St. \& Kingsessing Ave., Philadelphia, PA 19147

MR. RICHARD M. PENTA, Coordinator of For. Lang., 38 Normandy Rd., Lexington, MA 02173

MARIO P. PEREZ, Brevard College, Dir. of Lang. Lab., Brevard, NC 28712

D. L. PETHERBRIDGE, Assoc. Prof., University of Lethbridge, Lethbridge, Alberta, Canada

DR. DANIEL J. PETRIZZI, Coordinator For. Lang., Eisenhower College, Seneca Falls, NY 13148

JOHN A. PETTIT, Dept. of Mod. Lang., Marietta College, Marietta, OH 45750

J. E. PEYRAZAT, Lang. Lab. Dir., Roanoke College, Salem, VA 24153

MS CATHERINE J PHILLIPPON, 2727 Stuart, Berkeley, CA 94611 
SR. M. PHYLLIS, Notre Dame Preparatory, 815 Hampton Lane, Baltimore, MD 21204

GLADYS C. PIOLA, Dir. of Lang. Lab., 40 Owen Street, Apt. E II, Hartford, CT 06105

PROF. J. V. PLEASANTS, 20 Dewey Lane, Hampton Bays, NY 11946

WALTER PLUMB, Director, Instituto Anglo-Mexicano De Cultura, A. C., Maestro Antonio Caso 127, Mexico 4, D.F.

WILLIAM A. POPE, Dept. Mod. Lang., Keuka College, Keuka Park, NY 14478

PURDUE U. LIBRARIES, Serials Unit, Lafayette, IN 47907

U. OF QUEENSLAND, Main Library Serials Librarian, St. Lucia, Queensland 4067, Austraila

MR. T. QUINN, Dept. of French, Monash University, Clayton, Victoria 3168, Australia

$$
\text { - R - }
$$

PROF. LOUIS P. RAICHLE, 3 Shelterview Dr., Staten Island, NY 10304

JOHN A. RALLO, For. Lang. Dept., Darien HS, Darien, CT 06820

MISS RUTH RAWLINGS, Instr., Japan Nazarene College, P O Box 4, Yotsukaido, Imba Gun, Chiba Ken 284, Japan

WINSTON J. REESE, Lang. Lab. Dir., U. of Iowa, 125 Schaeffer Hall, Iowa City, IA 52240

PROF. DONA REEVES, Mod. Lang. Dept., Southwest Texas State U., Route 1, Box 239A, Buda, TX 78610

MRS FRANCES REID, Supr., Lang. Lab., Davidson College, Davidson, NC 28036

COL. HOWARD REINER, Ret., 37 Walnut Ave., Wheeling, W VA 26003

MRS. RUTH N. REMEIKA, Irvington HS, 70 Quabeck Ave., Irvington, NJ 07111

REVISTA COMUNICACOES E ARTES, Escola de Comunicacoes e Artes, Caixa Postal 8191, Sao Paulo, SP Brazil

CHARLES P. RICHARDSON, Dir. of Lang. Labs., Ellis Hall 26-A, Ohio University, Athens, OH 45701

RICHBOURG JR. HIGH SCHOOL LIBRARY, 500 Alabama Ave., Crestview, FL 32536

RIDGEWOOD HS, 627 East Ridgewood Ave, Att.: Mr. R. Tracy, Lang. Dept., Ridgewood, NJ 07451

PROF. W. M. RIVERS, Dept. of French, U. of Illinois, Urbana, IL 61801

MR. HENRY K. ROBERTS, ELEKTRON, D 6992 Weikersheim, Hartweg 6, Bundesrepublik, Deutschland

BEECHAM ROBINSON, Dir., Learning Center, U. of Wisconsin/Parkside, Kenosha, WI 53140

JANET D RODEWALD, Dir. Lang. Lab., Central Michigan U., Dept. of For. Lang., Mt Pleasant, MI 48858

DR. O. W. ROLFE, Dir. Lang. Lab, Dept. of For. Lang., U. of Montana, Missoula, MT 59801

E. ROSHGOLD, 22 Mishmeret Street, Afeka, Tel-Aviv, Israel 
ROSS MEMORIAL LIBRARY, Cottey College, Nevada, MO 64772

DR. R. ROY, Lang. Dept., School Brd. Office, 1577 Wall St. East, Winnipeg 3, Manitoba, Canada

MRS. LOIS P. RUSSELL, Consultant in Second Lang., New Brunswick Dept. of Educ., 71 Golf Club Rd., Fredericton, NB, Canada

\section{$-\mathbf{S}-$}

SADDLEBACK COLLEGE, 28000 Marguerite Pkwy., Mission Viejo, CA 92675

VICTOR SAMPON, Ch. For. Lang., Wayland Academy, Beaver Dam, WI 53916

SAN DIEGo STATE COLLEGE, Lang. Lab., 5402 College Ave., San Diego, CA 92115

SAN JOAQUIN DELTA COLLEGE, Zeller: For. Lang., 3301 Kensington Way, Stockton, CA 95204

MS MARY SANCHEZ, Supr. of For. Lang., 1960 East Druid Rd., PO Box 4688, Clearwater, FL 33518

MS ELEANOR L. SANDSTROM, For. Lang. Dir., R 305 Administration Bldg., Parkway at 21st St., Philadelphia, PA 19103

J. O. SAWYER, Dir. of Lang. Lab., U. of California, Berkeley, CA 94720

JESSE O. SAWYER, 1725 Grand View Dr., Berkeley, CA 94705

MARGARET SCARTH, Asst Prof. in Spanish, Erindale College, U. of Toronto, 3359 Mississauga Rd., Clarkson, Ontario, Canada

CARL W. SCHEFSKY, Instructional Media Dir., U. of Portland, 5000 North Willamette Blvd., Portland, OR 97203

EDWARD SCHEWE, 419 South Taylor Ave., Oak Park, IL 60302

VICTORIA SCHMITZ, Assoc. Dir., Schoonover Lab., Ohio Northern U., Ada, OH 45810

MS DIANNE SCHOBEL, 7801 Sagamore Dr., Cincinnati, OH 45236

D. SCHWENKER, Lang. Lab. Mgr., U. of Guelph, PO Box 129, Rockwood, Ontario, Canada

CHRM. LANG. DEPT., Scotch Plains-Fanwood HS, Westfield Rd, Scotch Plains, NJ 07076

RUSSELL H. SEARCH, Dir. of Audio Visual Mat., Bucknell U., Lewisburg, PA 17837

THE LIBRARIAN, Secondary Teachers College, Private Bag, Auckland, New Zealand

MARK W. SENG, Assoc. Prof., 2505 Princeton Dr., Austin, TX 78741

ALINE SEYDOUX, LRC Assoc. Dir., Loyola U., 7101 West 80th St., Los Angeles, CA 90045

MRS. MARY SHAPIRO, Assoc. Prof., Massachusetts State College, Bridge,water, MA 02324

MS JULIE SHEARER, 429-E Verbena Ct., Orlando, FL 32807

J. B. SHEEDY, Dir. Lang. Lab., PO Box 80 Congella, 72 Brand Rd., Durban, South Africa

WALTER A. SHEETS, Educational Service Center, 703 Washington St., Columbus, IN 47201

JOSEPH H. SHEEHAN, School of Lang. \& Ling., Georgetown U., Washington, DC 20007 
MARY D. SHERIDAN, Lang. Lab. Dir., Indian Hill Jr-Sr HS, 7370 Shawnee Rd., Apt. 3, Cincinnati, OH 45243

MARY M. SHIREY, 2419 Longview Ave. SW, Roanoke, VA 24014

LOREN W. SHORES, Chrm. For. Lang., Scarsdale HS, Scarsdale, NY 10583

SIERRA COLLEGE, Business Office, 5000 Rocklin Rd., Rocklin, CA 95677

MR. PETER M. SILINS, Asst. Prof. of German \& Russian, Wabash College, Crawfordsville, IN 47933

MR. LEON SILLS, Box 6892, Jersey City, NJ 07306

DOUGLAS J. SIMMONS, Asst. Prof. of For. Lang., Arizona State U., Tempe, AZ 85281

JOHN SIREVAAG, Head of German Dept., Augustana College, Rock Island, IL 61202

RUTH SKWAREK, Instructor, 15088 West Leonard Rd., Spring Lake, MI 49456

FRANCIS T. SMITH, Dir. Lang. Lab., Seton Hall U., South Orange, NJ 07079

SHANNON W. SMITH, 330 Mountain Ave., Bound Brook, NJ 08805

- WILLIAM FLINT SMITH, Dir. Lang. Lab., Purdue U., Dept. of Mod. Lang., Lafayette, IN 47907

WILLIAM J SMITHER, Prof. of Spanish, Tulane U., New Orleans, LA 70118

ELVIN V. SNYDER, Prof. of Spanish, Eastern Mennonite College, Harrisonburg, VA 22801

ALEX SOKALSKI, PO Box 48, New Haven, CT 06501

SOMERSET COUNTY COLLEGE, Serials Dept., PO Box 3300, Somerville, NJ 08876

SONAM Dept. c/o Mr. Masayori Sei, Intl. Mkt. Dept.Intl. Div., Sony Corp.PO Box 10, Tokyo Airport Post Office, Tokyo 149, Japan

PROF. WLLIAM SONANDRES, Chmn. For. Lang., Wilmington College, Box 1203, Wilmington, OH 45177

SONY CORP. OF AMERICA, Attn.: S. Koriyama, 4747 Van Dam St., Long Island City, NY 11101

LIBRARY, South Brunswick HS, Major Lane, Monmouth Junction, NJ 08852 SOUTHERN ILLINOIS U., Morris Library, Periodicals Recording Clerk, Carbondale, IL 62901

SPCK BOOKSTORE, 87 Union Ave., Salisbury, Rhodesia

O. R. SPINKS, Director, SUNY at Albany, Humanities B 16, Albany, NY 12203

ST. CLOUD STATE COLLEGE, Library Periodicals, St. Cloud, MN 56301

MS MARGARET E. STANDISH, (Chmn. Lang. Dept., Wayne Hills HS, Wayne, NJ 07444

STANFORD U. LIBRARIES, Order Dept., Stanford, CA 94305

ADRIAN L. STEINBERG, Dir. Lang. Lab., 'Temple U., College Hall 300, Philadelphia, PA 19122

ADLAI STEVENSON HS, Attn: Library Rt. 22, Prairie View, IL 60069

SUMMIT JUNIOR HS, Att.: Cyril Connelly, 272 Morris Ave., Summit, NJ 07901

LOIS M. SUTTON, Prof. of French, Baylor U., Waco, TX 70703 
RICHARD S. SWAIN, Tech. Dir., Marquette U., Mod. Lang. Lab., Milwaukee, WI 53233

S. A SWANSON, Lang Lab. Dir., Wisconsin State U., Oshkosh, WI 54901

MARTHA H SWIFT, Morgan Park Academy, 2153 W. 111th St., Chicago, IL 60643

DIR. DEPT. OF LANG. LABS, Level 2, Brennan Bldg., U. of Sydney, Sydney, New South Wales, Australia 2006

IVAN R. SYGODA, 227 Pelham Rd., DeWitt, NY 13214

GILBERT TAGGART, Assoc. Prof. of French, Dept. de Franc, Sir George Williams U., 1455 bd Maisonneuve, Montreal 107, PQ Canada

V. H. TALBOTT, TR Services Ltd., 287 MacPherson Ave., Toronto 7, Canada

MR. WALTER C. TAYLOR, Dept. of Spanish, Upsala College, East Orange, NJ 07019

TEACHERS COLLEGE LIBRARY, 525 West 120th St., New York, NY 10027

MR. GEORGE J. TELECKI, Harper \& Row Publ., Inc., 49 East 33rd St., New York, NY 10016

LIBRARY-Attn.: Serials, U. of Tennessee, Knoxville, TN 37916

THE U. OF TENNESSEE AT MARTIN, Rm 427 Humanities Bldg., Martin, TN 38237

STATE OF TEXAS, Dept. of For. Lang., Sam Houston State U., Huntsville, TX 77340

U. OF TEXAS, For. Lang. Educ. Cent. Library. 107 West 27th St., Austin, TX 78712

TEXAS U., Lang. Lab, Batts Hall, Austin, TX 78712

THE U. OF TEXAS AT EL PASO, Attn.: Dr. Past-Linguistics, El Paso, TX 79968

GERMANIC \& SLAVONIC LANGUAGES, Texas Tech. U., Lubbock, TX 79409

MR. JEAN R. THEUMA, For. Lang. Lab., 1890 East-West Rd., Honolulu, HI 96822

E. F. THORNE, Assoc Prof., West Liberty State College, West Liberty, W VA 26074

DR M. TIRADO, Chm. For. Lang. Dept., Wagner College, Staten Island, NY 10301

DR B. TOMKIN, St. Bonaventure U., St. Bonaventure, NY 14778

ADUN TOVEN, Asst. Prof., Pacific Lutheran U., Tacoma, WA 98447

ROBERT J. TRACY, Lang. Lab. Dir., Ridgewood HS, Ridgewood, NJ 07450

DR. MANFRED TRIESCH, Dir., German Center Boston, 170 Beacon St., Boston, MA 02138

MRS. GERTRUDE M. TRIPP, Lang. Lab. Dir., Elmhurst College, 190 Prospect Ave., Elmhurst, IL 60126

TURUN KIELI-INSTITUUTTI, Aurak 11, Turku, Finland

$-\mathrm{U}-$

MRS. CAROLYN M. UMMEL, Valley View HS, RR 2, Box 3370, Germantown, OH 45327

UNIVERSITY COLLEGE OF RHODESIA, Lang. Lab. No. 36943, PO Box MP167, Mt. Pleasant, Salisbury, Rhodesia 
E. C. VAN MERKENSTEIJN, Director, Lang. Lab., Logan Hall, U. of Pennsylvania, Philadelphia, PA 19104

GLENN J. VAN TREESE, Dir. Lang. Lab., Sweet Briar College, PO Box 18, Sweet Briar, VA 24595

GRACE R. VAN WALK, Grants Pass HS, 522 NE Olive, Grants Pass, OR 97526

DIRK B. VAN ZAANEN, Lang. Lab., State U. - Rutgers, 175 University Ave., Conklin Hall, Newark, NJ 07102

PROF. HUGH E. VELTMAN, Grand Valley State College, 615 Monroe Blvd., South Haven, MI 49090

LANG. LAB., Ventura College, 4667 Telegraph Rd., Ventura, CA 93003

GUNARS VEVERIS, For. Lang. Dept., Thomas More College, Box 85, Covington, KY 41017

LANG. LAB., U. of Virginia, 302 Cabell Hall, Charlottesville, VA 22903

\section{- W -}

HARRY A. WADE, Patrick Henry HS, 6702 Wandermere Dr., San Diego, CA 92120

SR MARIE ANDRE WALSH, IHM, St. Edward's U., Lang. Lab. Dir., 3001 South Congress, Austin, TX 78704

DR. HENRY WARKENTIN, Asst. Prof., For. Lang. Dept., Shippensburg State College, Shippensburg, PA 17257

DR. VIRGIL A. WARREN, 323 Corprew St., Fayette, MO 65248

WASHINGTON U. LIBRARIES, Serials/Acquisitions Dept., St. Louis, MO 63130

GEORGE WASHINGTON U., Romance Lang. \& Literatures, Washington, DC 20006

MR. JOHN A. WATSON, Dir. Lang. Lab., Virginia Union U., Richmond, VA 23220

DR. HARRY WEBB, Audio-Visual Dir., College of St. Thomas, St. Paul, MN 55101

DONALD B. WEBBER, Assoc. Prof. Lang. Lab., U. of the South, Sewanee, TN 37375

DR. ARNOLD L. WEINKAUF, Assoc Prof. of Lang., Michigan Technological U., Houghton, MI 49931

FOR. LANG. DEPT., Sr HS, 50 Rice St., Wellesley Hills, MA 02181

WEST TEXAS STATE U., Library, Box 748, W T STA, Canyon, TX 79015

U. OF WESTERN ONTARIO, General Library, Lawson Memorial Bldg., London, Ontario, Canada

MR. J. WHEELER, Education Faculty, Monash Univ., Clayton, Victoria, Australia

SIDNEY P. WHITE, Dept. Chrm., Morgan HS, Rt. 81, Clinton, CT 06413

KARL WIDMAIER, Mod. Lang. Instr., Atlantic Union College, S. Lancaster, MA 01561

PETER WIESE, Lang. Lab. Dir., Southern Connecticut State College, 501 Crescent St., New Haven, CT 06515 
MR. D. G. WILCOX, Public Relations, General Distributors Ltd., 1370 Sony Place, Fort Garry, Winnipeg, Canada

DR. GEORGE W. WILKINS, JR., Dir. Lang. Lab., Tulane U., New Orleans, LA 70118

CHARLES B. WILLIAMS, Dir. Lang. Lab., State University College, Sheldon Hall, Oswego, NY 13126

RAYMOND K. WILLIAMS, Dir. Lang. Lab., Fairleigh Dickinson U., Rutherford, NJ 07070

MRS. IONE WILLIS, RR 3, Milton, KY 40045

MS JACQUELINE WILLISON, Dir. Lang. Lab., Box 1524, Connecticut College, New London, CT 06320

LIBRARY, Wilton Sr. HS, 395 Danbury Rd., Wilton, CT 06897

JOE WIPF, 121 E. Knox Dr., West Lafayette, IN 47906

U. OF WISCONSIN AT MADISON, Library-Serials Dept., 728 State St., Madison, WI 53706

LIBRARY, Acquisitions Librarian, U. of Wisconsin/Milwaukee, 2311 E. Hartford, Milwaukee, WI 53201

WITTENBERG U., c/o J. B. Miller, Asst. Prof., Springfield, OH 45501

COLLEGE OF WOOSTER, Tech Services Division, The Andrews Library, Wooster, OH 44691

M. L. WORTHINGTON, Virginia State College, Box 428, Petersburg, VA 23803

MR. WALTER W. WYNN, 975 Ventura Ave., Albany, CA 94707

\section{$-\mathrm{X}-$}

XAVIER COLLEGE LIBRARY, c/o Sr. Rita M. MacInnes CND, Box 760, Sydney, Nova Scotia, Canada

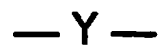

YORK U. LIBRARIES, Central Serials Record, 4700 Keele St., Downsview, Ontario, Canada

MR. GEORGE YOSHISHIGE, Leeward Community College, 96-045 Ala Ike, Pearl City, HA 96782

JAMES YZENBAARD, Dir. Lang. Labs., U. of Kentucky, School of Letters and Lang., Lexington, KY 40506

\section{$-\mathbf{Z}-$}

VALOIS A. ZARR, German Dept., East HS, 1134 South 17th East, Salt Lake City, UT 84108

ABT ZENTRALVERTRIEB, Fruchstr 3-4, DDR 1004, Berlin, German Dem. Republik

ZENTRUM FUR NEUE LERNVERFAHREN, Universitat, Tubingen, Federal Republic of Germany

PROF. ERNESTO ZIERER, Dept. de Idiomas y Linguistica, Apartado 315, Universidad Nacional de Trujillo, Trujillo, Peru

SISTER DOROTHY ZIMMERMAN, Dir. Lang. Lab., Mt. Mary College, Milwaukee, WI 53222

STEPHEN ZIRKO, For. Lang. Dir., Eastern Montana College, 1312 Crawford Dr., Billings, MO 59102 